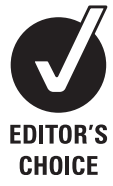

Pain and Anaesthesia Research Centre, Boyle's Department of Anaesthesia \& Pain Medicine, St Bartholomew's Hospital, London, UK

\section{Correspondence to}

Dr Vivek Mehta, Pain and Anaesthesia Research Centre

Boyle's Department of

Anaesthesia \& Pain Medicine, St Bartholomew's Hospital, London EC1A 7BE, UK;

vivek.mehta@mac.com

Received 5 July 2011

Accepted 8 October 2011

Published Online First

3 November 2011

\title{
Controversies and advances in non-steroidal anti-inflammatory drug (NSAID) analgesia in chronic pain management
}

\author{
Seema Shah, Vivek Mehta
}

\section{ABSTRACT}

Chronic pain can lead to significant disability with social and economic implications in the community. Traditional non-steroidal anti-inflammatory drugs (NSAIDs) have been part of the management of chronic pain. The risk of adverse events with traditional NSAIDs has led to the development of alternative therapeutic options. Differential blockade of the enzymes involved in pain and inflammation can offer therapeutic options without the gastrointestinal side effects. However, this may be at the expense of other major cardiovascular side effects. Pain pathways that involve peripheral transmission may be altered by local application of analgesia to the skin overlying the painful area. Recent guidelines for osteoarthritis treatment from the National Institute for Health and Clinical Excellence highlight the importance of topical NSAIDs in the armamentarium of pain management. NSAID combination drugs with gastric protection have provided alternatives to traditional NSAIDs, but the long term sequelae are unknown.

\section{INTRODUCTION}

Non-steroidal anti-inflammatory drugs (NSAIDs) are used in the treatment of acute pain for their opioid sparing effects, as part of a multimodal analgesic regimen. ${ }^{1}$ They have been gaining widespread usage in chronic pain, although this increase in prescription may be associated with significant morbidities. Gastrointestinal (GI) bleeding and ulceration, thrombotic events such as myocardial infarction and stroke, renal impairment, fluid retention and exacerbation of asthma are some of the side effects of NSAIDs.

The advances in NSAIDs have focused primarily on avoiding these potential side effects. These advances can be based either on targeting a new class of enzyme in the metabolic cascade, or the route of delivery of the medication, or the development of the newer combination therapy with proton pump inhibitors (PPIs). The GI side effect profile was in fact the driver of cyclo-oxygenase 2 (COX-2) targeted drugs in late 1990s. However, these medications soon fell into disrepute due to their potential effect on cardiovascular safety. Some of these drugs were subsequently withdrawn from prescribing amid concerns. The topical formulations of NSAIDs have been associated with fewer side effects and have been gaining widespread usage. NSAID combination drugs with PPI are being used for symptomatic treatment of chronic conditions in patients at risk of developing NSAID associated gastric ulceration. Newer formulations of these agents have recently been introduced into the market.

This review aims to outline the controversies associated with NSAIDs, provide an insight into the advances in NSAID use, and provide a framework for their rational and careful use with current guidelines.

\section{CONTROVERSIES WITH NSAIDS}

The traditional non-selective NSAIDs prescribed orally have been associated with widespread complications, of which GI bleeding and perforations have the most alarming figures. It has been reported that in the UK, of the 65000 hospital admissions a year for GI bleeding, 18000 are directly attributable to NSAIDs. ${ }^{2}$ Of these, there are 2230 deaths a year. In the community, a further 330 deaths are attributable to NSAIDs. This ranks GI mortality due to NSAID use higher than deaths due to asthma and cervical cancer in the UK. ${ }^{3-5}$ These deaths are iatrogenic and may be preventable.

A conservative estimate shows 107000 hospitalisations and 16500 NSAID related deaths occur among patients with rheumatoid arthritis (RA) or osteoarthritis (OA) every year in the USA. ${ }^{6}$ This is similar to the number of deaths from the AIDS and considerably greater than the number of deaths from multiple myeloma, asthma, cervical cancer, or Hodgkin's disease. If deaths from GI toxic effects from NSAIDs were tabulated separately in the National Vital Statistics reports, these effects would constitute the 15 th most common cause of death in the USA. ${ }^{7}$

A systematic review of studies published before 1997, and between 1997 and 2008, showed alarming figures too. ${ }^{8}$ Information was available for 61067 cases (81\% published since 1997) of which 5001 died. The mortality rate in all cases fell significantly, from $11.6 \%$ (95\% CI $11.0 \%$ to $12.2 \%$ ) in pre- 1997 studies to $7.4 \%$ (95\% CI $7.2 \%$ to $7.6 \%)$ in those published since 1997. In 5526 patients taking NSAID or aspirin, mortality increased from $14.7 \%$ (95\% CI $13.6 \%$ to $15.8 \%$ ) before 1997 to $20.9 \%$ (95\% CI $18.8 \%$ to $22.9 \%$ ) since 1997 . Data published since 1997 suggest that mortality in patients suffering from an upper GI bleed or perforation has fallen to one in 13 overall, but it remains higher at about one in five in those exposed to NSAID or aspirin.

This led to the development of COX-2 selective inhibitors in the 1990s to improve the GI safety profile for long term NSAID therapy. However, they have fallen into disrepute following major concerns about their safety profile. Rofecoxib was 
withdrawn voluntarily worldwide due to cardiovascular issues. ${ }^{9} 10$ The evidence available so far suggests that a higher cardiovascular risk may be associated with rofecoxib, while a lower cardiovascular risk may be associated with celecoxib. Valdecoxib's marketing in the USA and EU was suspended in 2005 due to serious cutaneous adverse reactions. ${ }^{9}{ }^{10}$ In 2008 lumiracoxib was withdrawn from the UK market, following concerns about liver toxicity associated with high doses. As further safety data will emerge, prescribers should be aware of the Summaries of Product Characteristics.

All NSAIDs have the propensity to cause fluid retention and can aggravate hypertension, although for certain agents, such as etoricoxib, this effect appears to be larger. Increasingly a prothrombotic risk (including myocardial infarction and stroke) has been identified with COX-2 selective agents in long term studies, and there does seem to be some evidence for a dose effect. ${ }^{11-14}$ These studies also demonstrate an increased cardiovascular risk from older agents such as diclofenac, which has high COX-2 selectivity. It is possible that naproxen does not increase prothrombotic risk $1 .{ }^{14}{ }^{15}$ All NSAIDs may antagonise the cardioprotective effects of aspirin.

\section{ADVANCES IN NSAID ANALGESIA COX-2 selective inhibitors}

In the 1990s, the evidence for two isoenzymes, COX-1 and COX-2, with different distribution, activity and effects led to the hypothesis that differential blockade of the two COX enzymes may broadly separate the analgesic and anti-inflammatory effects from the undesired effects resulting from COX-1 blockade. Driven by the desire for improved GI safety in long term NSAID therapy, the COX-2 selective inhibitors were developed. However, as already discussed, serious concerns pertaining to their safety profile has led to considerable debate and withdrawal of some of the drugs. Recommendations and strict guidelines have been implemented for the use of the remaining available COX-2 inhibitors.

Celecoxib and eterocoxib are available in the UK. They are licensed for the relief of pain in OA, RA, and ankylosing spondylitis. Parecoxib is the only intravenous formulation available, but is used in acute pain rather than chronic pain. The selective inhibitors of COX-2 are contraindicated in patients with ischaemic heart disease, cerebrovascular disease, peripheral arterial disease, and moderate or severe heart failure. They should be used with caution in patients with a history of left ventricular dysfunction, hypertension, oedema for any reason, and risk factors for heart disease.

There are some data to support the proposal that certain COX-2 selective agents reduce the incidence of serious GI adverse events (such as perforations, ulcers, and bleeds) when compared with less selective agents. Celecoxib and eterocoxib are highly selective agents. Dyspepsia, one of the most common reasons for discontinuation, remains a problem with all NSAIDs irrespective of COX-2 selectivity.

\section{Topical NSAIDs}

In the community, NSAIDs can be prescribed orally or topically, the latter route gaining more popularity due to its greater tolerability and limited side effect profile.

Topical NSAIS applications, which go directly onto the skin at the affected site using a rub-on solution, gel or adhesive skin patch, offer obvious theoretical advantages by minimising the systemic complications while expanding analgesic options. They have a relatively low bioavailability compared with oral NSAIDs and this may account for their favourable safety profile. Topical patches provide a fixed constant dose and local action. Gel formulations can be used on parts of the body that are not easily accessible by a patch, such as the fingers. Side effects tend to be localised to the site of application, such as itching and rashes.

So far, however, topical NSAIDs, also known as 'targeted peripheral therapy', have been slow to gain US Food and Drug Administration (FDA) approval or find their niche in the US pharmaceutical market. In Europe, however, where topical NSAIDs are more widely accepted, often in over-the-counter formulations, they have gained a place in treatment guidelines for OA of the knees, hips, and hands.

Topical NSAIDs are used to treat OA. After topical application, therapeutic levels of NSAIDs can be demonstrated in synovial fluid, muscles, and fasciae. They may have their pharmacological effects on both intra- and extra-articular structures. ${ }^{16-18}$ It is assumed that their mechanism of action is similar to that of oral NSAIDs; however, topical NSAIDs produce a maximal plasma NSAID concentration of only $15 \%$ of that achieved following oral administration of a similar dose. $^{16} 19$ Thus, it would be expected that topical NSAIDs would have far fewer systemic side effects than oral NSAIDs. Even if their pain relieving effect is less than that of oral NSAIDs, they may be an attractive option for the treatment of OA because they will produce fewer NSAID related adverse effects. It is possible that the act of rubbing and expectation of benefit may also contribute to any therapeutic effect from topical preparations. ${ }^{20-22}$

The Topical or Oral Ibuprofen study published by the National Institute for Health Research Health Technology Assessment programme in 2008 looked at topical or oral ibuprofen for chronic knee pain in older people. ${ }^{23}$ It recruited 585 people aged 50 or over from 26 general practices in the Medical Research Council General Practice Research Framework across the UK, to compare the clinical and cost effectiveness of oral with topical NSAIDs for the treatment of chronic knee pain. They also looked at patients' preferences and their attitudes towards the adverse side effects associated with the treatments, as this can influence perception on the effectiveness of the medication. The results showed that topical NSAIDs have an equivalent effect to oral NSAIDs on chronic knee pain in older people, and that those treated with oral preparations had more minor adverse effects such as indigestion, increased blood pressure, or worsening asthma. However, participants with more severe widespread pain preferred oral rather than topical medication, as they believed it might help other areas of pain while circulating around the body. Also, those using topical NSAIDs were more likely than patients taking oral NSAIDs to report severe chronic pain 3 months into treatment and to discontinue the treatment for lack of efficacy.

Before compiling their guidelines, the National Institute for Health and Clinical Excellence (NICE) looked at studies that investigated the efficacy and safety of topical NSAIDs compared with oral NSAIDs or placebo with respect to symptoms, function, and quality of life in adults with $\mathrm{OA} .{ }^{16}$ Two systematic reviews ${ }^{17} 24$ and two additional randomised controlled trials (RCTs) ${ }^{25} 26$ on topical NSAIDs were appraised.

The first meta-analysis included 13 RCTs (with $\mathrm{N}=1983$ participants) that focused on comparisons between topical NSAIDs versus placebo or oral NSAIDs in patients with OA. ${ }^{17}$ All RCTs were randomised and double blind. Studies included in the analysis differed with respect to OA site (eight RCTs knee OA; three RCTs hand OA; one RCT hip, knee and hand OA; one RCT hip and knee OA), type of topical NSAID used, type of oral NSAID used, treatment regimen and trial design (two RCTs 
crossover; 11 RCTs parallel group studies), size and length. The second meta-analysis included four RCTs (with $\mathrm{N}=1412$ participants) that focused on comparisons between topical diclofenac versus placebo or oral diclofenac in patients with knee $\mathrm{OA}^{24}$ All RCTs were randomised, double blind, parallel group studies.

A Cochrane review in 2010 of 47 double blinded RCTs showed that topical diclofenac, ibuprofen, ketoprofen, and piroxicam are significantly more effective and as safe as topical placebo in the treatment of acute musculoskeletal pain. ${ }^{27}$ The review did not address the safety of chronic use. Topical indomethacin and benzydamide were not significantly more effective than topical placebo; $6.3 \%$ of NSAID recipients experienced mild and transient local skin reactions, not significantly more than the $5.9 \%$ of placebo recipients who experienced these adverse events.

The European Medicines Agency's Committee for Medicinal Products for Human Use (CHMP) conducted a scientific review of topical ketoprofen following reports of photosensitivity reactions. ${ }^{28}$ It maintained that their benefit/risk profile remained favourable; however, patients should be reminded of photosensitivity measures such as handwashing after application and protecting treated areas from sunlight.

A number of these studies, mainly of knee OA, have shown short term ( $<4$ weeks) benefits from topical NSAID gels, creams, and ointments when compared with placebo. There are limited data on their long term effectiveness when compared with placebo. There are limited studies comparing other topical gels, creams, and ointments with oral NSAIDs.

Data from most of the studies have demonstrated a reduction in non-serious adverse effects when compared with oral NSAIDs, although topical preparations may produce local skin irritation. It seems logical that there would be a reduced risk given that the total dose of NSAIDs from topical application to one joint area is much less than when used orally. Thus, since there are some data supporting the effectiveness of topical NSAIDs, they are likely to be preferred to oral NSAIDs as early treatment for OA, particularly for patients who do not have widespread painful OA. So far, there are no data comparing topical NSAIDs with paracetamol or on the comparative risk and benefits from the long term use of oral or topical NSAIDs. Most of the clinical evidence is for the preparation of diclofenac in dimethylsulfoxide (DMSO).

Topical treatments are used in self-management, which helps change health behaviour positively. Often, people with OA will use the topical treatment on top of daily paracetamol and exercise to cope with flare-ups. This is in line with the evidence, which shows short term benefit. As a safe pharmaceutical option, topical NSAIDs are regarded as one of the second line options for symptom relief after the core treatments. They have therefore been placed on an equal footing with paracetamol.

NICE recommends healthcare professionals should consider offering topical NSAIDs for pain relief in addition to core treatment (education, exercise, and weight loss) for people with knee or hand OA. ${ }^{29}$ Topical NSAIDs and/or paracetamol should be considered ahead of oral NSAIDs, COX-2 inhibitors or opioids. Topical NSAIDs are relatively costly, but may be cost effective given that they prevent or delay use of oral NSAIDs with their associated serious adverse events.

Some of the commercially available topical preparations include Voltaren gel (a diclofenac topical), the Flector patch (contains diclofenac epolamine salt), Transfersome gel (contains ketoprofen), and Pennsiad (diclofenac). The http://clinicaltrials. gov/ website currently lists 19 trials of topical NSAIDs in various stages.

\section{Combination drugs with PPls}

There are two combination drugs available in the UK, both with naproxen. Napratec contains naproxen $(500 \mathrm{mg})$ and misoprostol $(200 \mathrm{mg})$, a synthetic prostaglandin $\mathrm{E}_{1}$ analogue which enhances several of the factors that maintain gastroduodenal mucosal integrity. The inclusion of misoprostol in the combination is to prevent naproxen induced gastric and duodenal ulceration. However, a higher starting dose of misoprostol-for prophylaxis against NSAID induced gastroduodenal ulceration - than that provided by Napratec may be required.

Vimovo has been approved by the FDA in the USA and has been recently licensed in the UK. It contains naproxen $(500 \mathrm{mg})$ and esomeprazole $(20 \mathrm{mg})^{30}$. It has been developed as a sequential delivery tablet combining an immediate release esomeprazole magnesium layer and an enteric coated delayed release core. As a result, esomeprazole is released in the stomach before the dissolution of naproxen in the small intestine. The enteric coating prevents naproxen release at $\mathrm{pH}$ levels $<5$, providing protection against local gastric toxicity of naproxen. Vimovo is currently licensed in the symptomatic treatment of OA, RA, and ankylosing spondylitis in patients who are at risk of developing NSAID associated gastroduodenal ulceration, and where treatment with lower doses of naproxen is not sufficient.

In two randomised, double blind, active controlled studies, the incidence of gastric and duodenal ulcers was significantly lower (5.6\%) after Vimovo treatment compared with enteric coated naproxen (23.7\%). Patients at risk of NSAID related gastric ulceration were included in the studies. Dyspepsia remains one of its most common side effects and may result in discontinuation.

\section{AVAILABLE RECOMMENDATIONS OF NSAID USAGE IN CHRONIC PAIN}

Although NSAIDs are used in almost all musculoskeletal chronic pain syndromes, most the evidence has primarily been limited to $\mathrm{OA}$ and RA. The NICE guidelines published in February 2008 for OA recommended that paracetamol and/or topical NSAIDs should be considered ahead of oral NSAIDs, COX-2 inhibitors or opioids. ${ }^{29}$ The guidelines stated that where paracetamol or topical NSAIDs are ineffective for pain relief for people with OA, then substitution with an oral NSAID/COX-2 inhibitor should be considered (box 1).

A large amount of clinical trial evidence that NICE reviewed supports the efficacy of both traditional NSAIDs and COX-2 selective agents in reducing the pain and stiffness of $O A$, with the majority of studies reflecting short term usage and involving knee or hip joint OA. All NSAIDs, irrespective of COX-1 and COX-2 selectivity, are associated with significant morbidity and mortality due to adverse effects on the GI, renal, and cardiovascular systems. It should be further noted that although supra-normal doses of newer agents are commonly used in clinical trials in order to demonstrate safety, the clinical trials usually recruit patients without any serious comorbidities. This is in contrast to the routine population who would have those comorbidities.

The NICE recommendations for RA from 2009 suggest that although NSAIDs may provide symptomatic benefit, they do not modify the course of the disease. ${ }^{31}$ The GI risks of NSAIDs in RA are similar to OA, but the cardiovascular risks are higher in RA. It is recommended to prescribe the lowest effective dose over a short period of time-that is, 'when necessary', not regularly. There is an additional benefit in co-prescribing a PPI. Topical NSAID gels have a limited role in RA, due to the polyarticular nature of the disease. 


\section{Box 1 NICE recommendations}

- Where paracetamol or topical NSAIDs provide insufficient pain relief for people with osteoarthritis, then the addition of an oral NSAID/COX-2 inhibitor to paracetamol should be considered.

- Oral NSAIDs/COX-2 inhibitors should be used at the lowest effective dose for the shortest possible period of time.

- When offering treatment with an oral NSAID/COX-2 inhibitor, the first choice should be either a standard NSAID or a COX-2 inhibitor (other than etoricoxib $60 \mathrm{mg}$ ). In either case, these should be co-prescribed with a proton pump inhibitor (PPI), choosing the one with the lowest acquisition cost.

- All oral NSAIDs/COX-2 inhibitors have analgesic effects of a similar magnitude but vary in their potential gastrointestinal, liver, and cardiorenal toxicity; therefore when choosing the agent and dose, healthcare professionals should take into account individual patient risk factors, including age. When prescribing these drugs, consideration should be given to appropriate assessment and/or ongoing monitoring of these risk factors.

- If a person with $\mathrm{OA}$ needs to take low dose aspirin, healthcare professionals should consider other analgesics before substituting or adding an NSAID or COX-2 inhibitor (with a PPI) if pain relief is ineffective or insufficient.

The European Medicines Agency concurred with NICE in recommending that doctors should use the lowest effective dose of the COX-2 medicine for the shortest possible duration of treatment. ${ }^{9}$ They also recommended that these medicines should not be used in patients with ischaemic heart disease or stroke, and etoricoxib should additionally be contraindicated in patients with hypertension whose blood pressure is not yet under control. Prescribers should exercise caution when using COX-2 inhibitors in patients with risk factors for heart disease such as hypertension, hyperlipidaemia, diabetes mellitus, and smoking, or peripheral arterial disease.

The European Medicines Agency guidelines in 2006 for nonselective NSAIDs emphasised that non-selective NSAIDs are important treatments for arthritis and other painful conditions. ${ }^{32}$ While it cannot be excluded that non-selective NSAIDs may be associated with a small increase in the absolute risk for thrombotic events, especially when used at high doses for longterm treatment, the overall benefit/risk balance remains favourable when used in accordance with the product infor-

\section{Main messages}

Although NSAIDs are used in the management of chronic pain, they can have serious complications.

- NSAIDs should be used for the shortest possible period of time at the lowest effective dose.

- Topical formulations have the potential to provide valuable analgesia with a reduced side effect profile with short term use.

- Long term risks and benefits of topical NSAIDs have not been assessed yet, and it is important to remember that they too contain black box warnings regarding risks of thrombotic events and gastrointestinal effects.

\section{Current research questions}

- What is the evidence for efficacy of topical NSAIDs in other chronic conditions apart from osteoarthritis and rheumatoid arthritis?

- Are topical NSAIDS as effective or more effective than paracetamol alone?

- What are the comparative data within different classes of drugs, as all the current studies are placebo controlled studies?

- Is there genetic variation in NSAID induced analgesia?

- What are the long term effects of proton pump inhibitor combination drugs, such as Vimovo?

mation. Patients and prescribers should use NSAIDs as necessary at the lowest effective dose for the shortest possible duration to control symptoms.

In February 2007, the American Heart Association recommended that doctors treating chronic pain in patients with or at risk for heart disease consider NSAIDs as a last line of treatment. ${ }^{33}$

\section{CURRENT RESEARCH GAPS}

The short term use and benefits of topical NSAIDs have been reflected in the NICE guidelines for the treatment of OA. The evidence with regards to efficacy of these drugs is predominantly limited to the OA and RA populations. Their efficacy needs to be further investigated in general musculoskeletal and chronic pain conditions.

There are no conclusive data on the risks and benefits of long term use (>12 months) of topical preparations of NSAIDs. There is a lack of comparative studies within the classes of drugs-for example, within the topical group and within the PPI combination group. The data are predominantly limited to placebo controlled trials. There are also no data comparing topical NSAIDs to paracetamol alone.

The trials of the PPI combination drugs, namely Vimovo, looked at complications over a period of 12 months. The safety profile over a longer period needs to be studied.

\section{Key references}

- National Institute for Health and Clinical Excellence. Osteoarthritis: The Care and Management of Osteoarthritis In Adults. NICE clinical guideline 59. http://www.nice.org.uk/ nicemedia/pdf/CG59NICEguideline.pdf (accessed 28 Aug 2010).

- EMEA Press release. European Medicines Agency concludes action on COX-2 inhibitors. EMEA Doc. Ref. EMEA/207766/ 2005.

- Moore R, Tramer M, Carroll D, et al. Quantitative systematic review of topically applied non-steroidal anti-inflammatory drugs. BMJ 1998;316:333-8.

- Garcia Rodriguez L, Jick H. Risk of upper gastrointestinal bleeding and perforation associated with individual nonsteroidal anti-inflammatory drugs. Lancet 1994;343:769-71.

- Graham DJ, Campen D, Hiu R, et al. Risk of acute myocardial infarction and sudden cardiac death in patients treated with cyclo-oxygenase 2 selective and non-selective non-steroidal anti-inflammatory drugs: nested case-control study. Lancet 2005;365:475-8. 
There may be some genetic basis for the variation in NSAID induced analgesia. To date, there has been no evidence for this. Knowledge from this may permit a highly individualised analgesic prescription.

\section{SUMMARY}

Despite the long and well established place of NSAIDs in treating chronic pain, their role requires continuing evaluation with respect to safety. The development of topical NSAIDs and the newer fixed dose preparations with PPI may provide symptomatic relief, effectively potentially avoiding the GI and cardiovascular side effects of oral NSAIDs. This increases the therapeutic options available for chronic pain management, given concerns about long term opioid use and COX-2 inhibitors.

\section{MULTIPLE CHOICE QUESTIONS (TRUE (T)/FALSE (F); ANSWERS AFTER THE REFERENCES)}

1. Concerning the management of chronic pain:

A. A multimodal approach using NSAIDs will have an opioid sparing effect

B. NSAIDs should be used as a first line management

C. Pharmacological treatment should be used in isolation

D. NSAIDs have been shown to be safe for long term use

\section{Concerning the use of oral NSAIDs:}

A. The gastrointestinal side effects are negligible and do not have an impact on mortality rates

B. COX-2 inhibitors can increase the risk of myocardial infarctions and stroke

C. NSAIDs do not worsen existing hypertension when prescribed in normal doses

D. Oral NSAIDs and COX-2 inhibitors can vary in their potential to cause cardiovascular, gastrointestinal, renal, and liver toxicity

\section{Concerning the current recommendations in NSAID prescription:}

A. Oral NSAIDs/COX-2 inhibitors should be prescribed at high doses for short periods of time

B. Paracetamol or topical NSAIDs should be used as first line pharmacological management for treatment of pain from osteoarthritis

C. Topical NSAIDs have a major role in the treatment of pain from rheumatoid arthritis

D. The European Medicines Agency recommend that COX-2 inhibitors should not be prescribed at all in patients with risk factors for heart disease such as hypertension and diabetes

\section{Concerning the recent advances in NSAID use:}

A. Topical NSAIDs have the same bioavailability as oral NSAIDs

B. Side effects of topical NSAIDs tend to be minor cutaneous irritation

C. Topical NSAIDs are in widespread use both in Europe and the USA

D. There are national guidelines concerning the use of NSAIDs in the treatment of osteoarthritis

\section{Concerning the use of topical NSAIDs}

A. Topical NSAIDS have been shown to be effective for long term use

B. Topical NSAIDs still carry a risk of thrombotic events and gastrointestinal side effects
C. Topical NSAIDs should be used in conjunction with exercise and weight loss for patients with osteoarthritis

D. There is no need for more clinical research to be done in the safety and effectiveness of topical NSAIDs

Competing interests None.

Provenance and peer review Not commissioned; internally peer reviewed.

\section{REFERENCES}

1. Hyllested M, Jones S, Pederson JL, et al. Comparative effect of paracetamol, NSAIDs or their combination in postoperative pain management: a qualitative review. Br J Anaesth 2002;88:199-214.

2. Blower A, Brooks A, Fenn G, et al. Emergency admissions for upper gastrointestina disease and their relation to NSAID use. Aliment Pharmacol Ther 1997;11:283-91.

3. Tramer M, Moore R, Reynolds J, et al. Quantitative estimation of rare adverse events which follow a biological progression: a new model applied to chronic NSAID use. Pain 2000;85:169-82.

4. Office for National Statistics. 1997. http://www.statistics.gov.uk/statbase/ssdataset. asp?vInk=6922 (accessed 25 Aug 2010).

5. Moore R, Phillips C. Cost of NSAID adverse effects to the UK National Health Service. J Med Econom 1999:2:45-55.

6. Singh G, Triadafilopoulos G. Epidemiology of NSAID-induced Gl complications. J Rheumatol 1999;26:18-24.

7. National Centre for Health Statistics. National Vital Statistics Report. Vol. 58. 1997. http://www.cdc.gov/NCHS/data/nvsr/nvsr58/nvsr58_19.pdf laccessed 3 Sep 2010)

8. Straube S, Tramer M, Moore R, et al. Mortality with upper gastrointestinal bleeding and perforation: effects of time and NSAID use. BMC Gastroenterol 2009;9:14.

9. EMEA Press release. European Medicines Agency Concludes Action on COX-2 Inhibitors. London, Uk: EMEA, 2005. Doc. Ref. EMEA/207766/2005.

10. Food and Drug Administration. FDA Announces Series of Changes to the Class of Marketed Non-Steroidal Anti-Inflammatory Drugs (NSAIDs). Rockville, MD: FDA, 2005.

11. Bresalier R, Sandler R, Quan H, et al. Cardiovascular events associated with rofecoxib in a colorectal adenoma chemoprevention trial. N Engl J Med 2005;352:1092-102.

12. Graham D, Campen D, Hiu R, et al. Risk of acute myocardial infarction and sudden cardiac death in patients treated with cyclo-oxygenase 2 selective and non-selective anti-inflammatory drugs: nested case-control study. Lancet 2005;365:475-81.

13. Solomon S, McMurray J, Pfeffer $\mathrm{M}$, et al. Cardiovascular risk associated with celecoxib in a clinical trial for colorectal adenoma prevention. $N$ Engl J Med 2005;352:1071-80

14. Langford R, Mehta V. Selective cyclooxygenase inhibition: its role in pain and anaesthesia. Biomed Pharmacother 2006;60:323-8.

15. Bombardier C, Laine I, Reicin A, et al. Comparison of upper gastrointestinal toxicity of rofecoxib and naproxen in patients with rheumatoid arthritis. VIGOR Study Group. N Engl J Med 2000;343:1520-8.

16. Dominkus $\mathbf{M}$, Nicolakis $\mathrm{M}$, Kotz $\mathrm{R}$, et al. Comparison of tissue and plasma levels of ibuprofen after oral and topical administration. Arzneimittelforschung 1996;46:1138-43.

17. Lin J, Zhang W, Jones A, et al. Efficacy of topical non-steroidal anti-inflammatory drugs in the treatment of osteoarthritis: meta-analysis of randomized controlled trials. BMJ 2004;329:324.

18. Rolf C, Engstrom B, Beauchard C et al Intra-articular absorption and distribution of ketoprofen after topical plaster application and oral intake in 100 patients undergoing knee arthroscopy. Rheumatology (Oxford) 1999;38:564-7.

19. Heyneman C, Lawless-Liday C, Wall G. Oral versus topical NSAIDs in rheumatic diseases: a comparison. Drugs 2000;60:555-74.

20. Quandt S, Chen $\mathrm{H}$, Grzywacz J, et al. Use of complementary and alternative medicine by persons with arthritis: results of the National Health Interview Survey. Arthritis Rheum 2005;53:748-55.

21. Rouster-Stevens K, Nageswaran S, Arcury T, et al. How do parents of children with juvenile idiopathic arthritis (JIA) perceive their therapies? BMC Complement Altern Med 2008;8:25

22. Vaile J, Davis P. Topical NSAIDs for musculoskeletal conditions: a review of the literature. Drugs 1998;56:783-79.

23. Underwood M, Ashby D, Carnes D, et al. Topical or Oral ibuprofen for Chronic Knee Pain in Older People. The TOIB Study Executive Summary. Vol. 12. Health Technology Assessment, 2008. http://www.hta.ac.uk/pdfexecs/summ1222.pdf laccessed 15 Sep 2010).

24. Towheed T. Pennsaid therapy for osteoarthritis of the knee: a systematic review and metaanalysis of randomized controlled trials. J Rheumatol 2006;33:567-73.

25. Niethard F, Gold M, Solomon G, et al. Efficacy of topical diclofenac diethylamine ge in osteoarthritis of the knee. J Rheumatol 2005;32:2384-92.

26. Trnavsky K, Fischer M, Vogtle-Junkert U, et al. Efficacy and Safety of $5 \%$ Ibuprofen Cream Treatment in Knee OA. Results of a Randomized, Double-Blind, PlaceboControlled Study. J Rheumatol 2004;31:565-72.

27. Massey T, Derry S, Moore R, et al. Topical NSAIDS for acute pain in adults. Cochrane Database Syst Rev 2010;(6):CD007402. http://onlinelibrary.wiley.com/o/ cochrane/clsysrev/articles/CD007402/frame.html (accessed 6 Dec 2010). 
28. EMEA Press Release. European Medicines Agency Confirms Positive Benefit-risk Balance of Topical Formulations of Ketoprofen. London, UK: EMEA, 2010. Doc Ref EMA/CHMP/465633/2010.

29. National Institute for Health and Clinical Excellence. Osteoarthritis: The Care and Management of Osteoarthritis in adults. NICE clinical guideline 59. http://www. nice.org.uk/nicemedia/pdf/CG59NICEguideline.pdf (accessed 28 Aug 2010).

30. Sostek M, Fort J, Estboen L, et al. Evaluation of the long-term safety of a fixed-dose combination of naproxen and esomeprazole magnesium in patients requiring chronic nonsteroidal anti-inflammatory drug (NSAID) therapy: results from a 12-month multicenter safety study. Curr Med Res Opin 2011;27:847-54.

31. National Institute for Health and Clinical Excellence. Rheumatoid Arthritis. National Clinical Guideline for Management and Treatment in Adults. NICE clinical guideline 79. http://www.nice.org.uk/nicemedia/live/12131/43326/43326.pdf (accessed 24 Jan 2011).

32. EMEA Press Release. Cardiovascular Safety Data on Non-selective NSAIDs. EMEA. Doc Ref EMEA/410862/2006.
33. American Heart Association/American College of Cardiology Joint Scientific Statement. 2007. http://www.newsroom.heart.org/index.php?s=438item =112 (accessed 28 Aug 2010).

\section{ANSWERS}

1. $A$ (T): B (F): C (F): D (F)

2. $A(F) ; B(T) ; C(F) ; D(T)$

3. A (F): B (T): C (F): D (F)

4. A (F); B (T); C (F); D (T)

5. A (F); $B(T) ; C(T) ; D(F)$

\section{Postgraduate Medical Journal alerts}

Sign up for our electronic table of contents alert and you will never miss new issues of Postgraduate Medical Journal when published online. Stay ahead and up to date by visiting pmj.bmj.com. 